\title{
Perspectives given by structural connectivity bridge the gap between structure and function
}

\author{
Hiromasa Takemura ${ }^{1,2} \cdot$ Michel Thiebaut de Schotten $^{3,4}$
}

Published online: 15 May 2020

(c) The Author(s) 2020

\begin{abstract}
"Afferent, efferent and intrinsic connections, as well as cell types and their properties, are the structural basis of a brain region's function" (Zilles and Amunts 2015). Over the last several decades, neuroscience has made enormous progress in understanding brain-function mechanisms at different spatial scales, ranging from the single-neuron level to macroscale cortical maps. While some reports demonstrate that a function can be localised into a specific area (localisationism), a collection of neuroscience studies also indicate that functions are mediated through the interaction of multiple brain areas. We will argue that extreme localisationism thinking has lost perspective. While areas can be sensitive to specific functions, they are not independently processing the information. For instance, reading this text requires the involvement of a system of interconnected brain areas analysing visual words and phonological and lexical information (Wandell et al. 2012). Hence, there is a pressing need to understand 'structural connectivity', which is a term generally referring to anatomical connections between brain areas. Structural connectivity is essential in understanding the circuitry supporting the interaction between brain areas and in bridging anatomy with function.
\end{abstract}

Hiromasa Takemura

htakemur@nict.go.jp

Michel Thiebaut de Schotten

michel.thiebaut@gmail.com

1 Center for Information and Neural Networks (CiNet), National Institute of Information and Communications Technology, and Osaka University, Suita-shi, Japan

2 Graduate School of Frontier Biosciences, Osaka University, Suita-shi, Japan

3 Brain Connectivity and Behaviour Laboratory, Sorbonne Universities, Paris, France

4 Groupe D'Imagerie Neurofonctionnelle, Institut Des Maladies Neurodégénératives-UMR 5293, CNRS, CEA University of Bordeaux, Bordeaux, France
Despite many connectomics projects (Bakker et al. 2012; Burns et al. 2013; Van Essen et al. 2013; Zingg et al. 2014; Oh et al. 2014; Majka et al. 2020), a comprehensive understanding of structural connectivity of the human brain is still missing. While several methods for studying structural connectivity have been developed, they all present a tradeoff between advantages and limitations. For instance, higher spatial resolution comes with a smaller field of view, while other methods, typically diffusion magnetic resonance imaging (dMRI), have a lower resolution but cover the whole brain. Similarly, some methods are only applicable to ex vivo animal brains, while other methods are available for living human brains. A consensus on the whole picture of the structural connectivity is challenged by these limitations (Rushmore et al. 2020). 'Structural connectivity' is also a multidimensional concept that is far from the simplified notion of 'connected' or 'not connected'. At a cellular/ molecular level, the type of synapses will impact the underlying neural circuitry with quite different functional implications (Bargmann and Marder 2013). At a macroscopic level, there is increasing evidence from dMRI studies showing that differences in white matter bundles can explain behavioural diversity among human individuals (Catani et al. 2007; Thiebaut de Schotten et al. 2011; Huber et al. 2018; Oishi et al. 2018), as well as impact precision medicine (Forkel et al. 2014, 2020; Takemura et al. 2019; Forkel and Thiebaut de Schotten 2020).

This new Brain Structure and Function special issue entitled 'Structural connectivity of the cerebral cortex' aims at providing the reader with a comprehensive understanding of the organisation of the brain's structural connectivity, based on different methods (Lanciego and Wouterlood 2020; Blazquez Freches et al. 2020; Huang et al. 2020; Oishi et al. 2020; Kaneko et al. 2020; Woodward et al. 2020). The special issue also clarifies the multidimensional concept of structural connectivity by collecting articles that investigate how its variations at different scales affect brain functions (Rockland 2020; Gamberini et al. 2020; Andre et al. 2020; 
Ioannucci et al. 2020; Rooks et al. 2020). Finally, we invited investigators developing cutting-edge clinical applications of structural connectivity to provide an insight into how measuring this connectivity in the human brain can benefit society (Vanderweyen et al. 2020; David et al. 2020).

Typically, structural connectivity is derived from chemical tracers applied to non-human primate brains (Schmahmann and Pandya 2006). Since this original work, new types of tracers appeared with different properties that render interpretation difficult for non-experts. Furthermore, making raw tracer data machine-readable for quantitative analyses remains a challenge. Lanciego and Wouterlood (2020) (this issue) reviewed a wide range of neuroanatomical tracttracing methods, from classical to modern, and examined their advantages and disadvantages. Woodward et al. (2020) (this issue) also proposed a new tracer processing pipeline in marmoset brains. They succeeded at quantifying anterograde tracer signals by using the latest artificial intelligence algorithms. They included three-dimensional reconstructions of tracer and histological data, as well as registration to a standard brain space as an MRI dataset, which bridges the gap between tracer data and the aforementioned dMRI data.

Other studies in this special issue demonstrate that direct dMRI data from the living human brain can show its connectional organisation. Accordingly, Blazquez Freches et al. (2020) (this issue) used dMRI-based tractography to identify the essential principles of structural connectivity in the human temporal cortex. They identified three connectivity gradients that displayed, within the temporal cortex, distinct connections that were supported by different white matter tracts and showed specific relationships to functions. The brain can also be divided into subregions (i.e. parcellation) according to the areas they preferentially connect. These methods are often tricky to implement; therefore, Reuter et al. (2020) (this issue) provided open-source software that makes the connectivity-based parcellation method broadly accessible to the neuroscience community. dMRI can also estimate further spatial details of the white matter. For instance, Huang et al. (2020) (this issue) proposed a framework to determine the diameter of white matter axons in the living human brain. Oishi et al. (2020) (this issue) performed dMRI acquisitions on an ex vivo human brain with a high spatial resolution to characterise the white matter organisation in the subthalamic area. Finally, Kaneko et al. (2020) (this issue) used high spatial resolution dMRI data from the common marmoset to investigate occipital white matter tracts and clarify the similarities and differences in the visual system fibre tracts across primate species. Hence, despite some considerable advances, these studies make clear that a comprehensive understanding of the white matter anatomy, particularly in humans, is still missing and crucially needed.

Knowledge derived from the anatomical organisation of white matter goes well beyond anatomy, as it contributes to the understanding of functional circuitry and cortical dynamics (Rockland 2020, this issue). For instance, singleaxon analyses have revealed essential organisation principles of thalamocortical or cortico-cortical connections, such as laminar organisation and the existence of axon collaterals and intrinsic/extrinsic connections. Specifically, differences in laminar specificity between feedforward and feedback connections, together with collaterals within and across areas, provide essential insights into the recurrent nature of cortical visual processing. Hence, the discovery of white matter principles offers critical information to interpret the neural dynamics of intra-areal communications, as well as the primary mechanisms supporting functions and pathologies.

In line with this statement, the review from Gamberini et al. (2020) (this issue) provides a comprehensive overview of the organisation of the superior parietal lobule (SPL), which combines cytoarchitecture, structural connectivity and electrophysiology together with functional MRI. The study indicates that while macaque SPL has often been considered to be entirely contained within Brodmann's area 5, it can be divided into several regions with distinct connections to somatosensory, motor, visual and frontal cortices. Importantly, functional differences among these areas may be associated with anatomical connectivity differences, such as the degree of neuronal modulation by visual inputs or involvement in the control of limb movement. Another example of function being tightly linked with structural connections includes the relationship between the microstructural properties of white matter tracts in the limbic system and the subclinical diversity of internal and external behaviour among children and adolescents (Andre et al. 2020) (this issue). Similarly, the lateralisation of the limbic tracts shown in Ioannucci et al. (2020) (this issue) is related to the asymmetrical facial expression of happiness and sadness. Finally, the connectivity matrices derived from dMRI data demonstrate some fair differences according to everyday decisionmaking capacity in older age (Rooks et al. 2020) (this issue). These examples highlight the importance of white matter connectivity to the functional organisation of the brain and the differences in behaviour observed.

A better understanding of structural connectivity in humans can also impact our society. David et al. (2020) (this issue) discovered a relationship between the tissue property of specific fibre tracts and aggressive behaviours after military deployment, which may help with the early identification of soldiers who need an intervention procedure. Vanderweyen et al. (2020) (this issue) reviewed the role of $\mathrm{dMRI}$ in the optimisation of neurosurgical resection strategy and outcome. These papers provide a perspective on promising future applications to address contemporary problems outside academia. 
While this special issue covered a wide range of topics and methods, it does not include other promising approaches including polarised light imaging (Axer et al. 2011; Wang et al. 2018; Caspers and Axer 2019), tissue clearing methods (Hama et al. 2011; Chung and Deisseroth 2013), and expansion microscopy (Wassie et al. 2019). As these measurements rely on different principles and concepts, additional studies comparing these methods with ones presented here will be essential to link the different spatial scales and provide a comprehensive understanding of structural connectivity. Other avenues, such as how neural dynamics are related to the underlying structural connectivity, remain utterly open for future generations to explore. We wish that collaborative efforts among neuroanatomists with different disciplines will help address these difficult remaining issues in the next several decades.

Acknowledgements We are grateful to all authors contributing to this special issue. We are also grateful to Co-Editors-in-Chief Laszlo Zaborszky and Karl Zilles for their constant encouragement and scientific support, and to Neil Solomon for operational support. HT is supported by the Japan Society for the Promotion of Science (JSPS) KAKENHI (grant number, JP17H04684). MTS is funded by the European Research Council (ERC) under the European Union's Horizon 2020 research and innovation programme (Grant agreement No. 818521).

Open Access This article is licensed under a Creative Commons Attribution 4.0 International License, which permits use, sharing, adaptation, distribution and reproduction in any medium or format, as long as you give appropriate credit to the original author(s) and the source, provide a link to the Creative Commons licence, and indicate if changes were made. The images or other third party material in this article are included in the article's Creative Commons licence, unless indicated otherwise in a credit line to the material. If material is not included in the article's Creative Commons licence and your intended use is not permitted by statutory regulation or exceeds the permitted use, you will need to obtain permission directly from the copyright holder. To view a copy of this licence, visit http://creativecommons.org/licenses/by/4.0/.

\section{References}

Andre QR, Geeraert BL, Lebel C (2020) Brain structure and internalizing and externalizing behavior in typically developing children and adolescents. Brain Struct Funct 225(4):10. https://doi. org/10.1007/s00429-019-01973-y

Axer M, Amunts K, Grässel D et al (2011) A novel approach to the human connectome: ultra-high resolution mapping of fiber tracts in the brain. Neuroimage 54:1091-1101

Bakker R, Wachtler T, Diesmann M (2012) CoCoMac 2.0 and the future of tract-tracing databases. Front Neuroinform 6:30

Bargmann CI, Marder E (2013) From the connectome to brain function. Nat Methods 10:483-490

Blazquez Freches G, Haak KV, Bryant KL et al (2020) Principles of temporal association cortex organisation as revealed by connectivity gradients. Brain Struct Funct 225(4):16. https://doi. org/10.1007/s00429-020-02047-0
Burns R, Roncal WG, Kleissas D et al (2013) The open connectome project data cluster: scalable analysis and vision for high-throughput neuroscience. Int Conf Sci Stat Database Manag. https://doi. org/10.1145/2484838.2484870

Caspers S, Axer M (2019) Decoding the microstructural correlate of diffusion MRI. NMR Biomed 32:e3779

Catani M, Allin MPG, Husain M et al (2007) Symmetries in human brain language pathways correlate with verbal recall. Proc Natl Acad Sci U S A 104:17163-17168

Chung K, Deisseroth K (2013) CLARITY for mapping the nervous system. Nat Methods 10:508-513

David S, Heesink L, Geuze E et al (2020) Regions of white matter abnormalities in the arcuate fasciculus in veterans with anger and aggression problems. Brain Struct Funct 225(4):11. https://doi. org/10.1007/s00429-019-02016-2

Forkel SJ, Rogalski E, Drossinos Sancho N et al (2020) Anatomical evidence of an indirect pathway for word repetition. Neurology 94:e594-e606

Forkel SJ, Thiebaut de Schotten M (2020) Towards metabolic disconnection-symptom mapping. Brain 143:718-721

Forkel SJ, Thiebaut de Schotten M, Dell'Acqua F et al (2014) Anatomical predictors of aphasia recovery: a tractography study of bilateral perisylvian language networks. Brain 137:2027-2039

Gamberini M, Passarelli L, Fattori P, Galletti C (2020) Structural connectivity and functional properties of the macaque superior parietal lobule. Brain Struct Funct 225(4):19. https://doi.org/10.1007/ s00429-019-01976-9

Hama H, Kurokawa H, Kawano H et al (2011) Scale: a chemical approach for fluorescence imaging and reconstruction of transparent mouse brain. Nat Neurosci 14:1481-1488

Huang SY, Tian Q, Fan Q et al (2020) High-gradient diffusion MRI reveals distinct estimates of axon diameter index within different white matter tracts in the in vivo human brain. Brain Struct Funct 225(4):15. https://doi.org/10.1007/s00429-019-01961-2

Huber E, Donnelly PM, Rokem A, Yeatman JD (2018) Rapid and widespread white matter plasticity during an intensive reading intervention. Nat Commun 9:2260

Ioannucci S, George N, Friedrich P et al (2020) White matter correlates of hemi-face dominance in happy and sad expression. Brain Struct Funct 225(4):10. https://doi.org/10.1007/s00429-020-02040-7

Kaneko T, Takemura H, Pestilli F et al (2020) Spatial organization of occipital white matter tracts in the common marmoset. Brain Struct Funct 225(4):14. https://doi.org/10.1007/s00429-02002060-3

Lanciego JL, Wouterlood FG (2020) Neuroanatomical tract-tracing techniques that did go viral. Brain Struct Funct 225(4):32. https ://doi.org/10.1007/s00429-020-02041-6

Majka P, Bai S, Bakola S et al (2020) Open access resource for cellularresolution analyses of corticocortical connectivity in the marmoset monkey. Nat Commun 11:1133

Oh SW, Harris JA, Ng L et al (2014) A mesoscale connectome of the mouse brain. Nature 508:207-214

Oishi H, Takemura H, Aoki SC et al (2018) Microstructural properties of the vertical occipital fasciculus explain the variability in human stereoacuity. Proc Natl Acad Sci USA 115:12289-12294

Oishi K, Mori S, Troncoso JC, Lenz FA (2020) Mapping tracts in the human subthalamic area by $11.7 \mathrm{~T}$ ex vivo diffusion tensor imaging. Brain Struct Funct 225(4):20. https://doi.org/10.1007/s0042 9-020-02066-X

Reuter N, Genon S, Kharabian Masouleh S et al (2020) CBPtools: a Python package for regional connectivity-based parcellation. Brain Struct Funct 225(4):15. https://doi.org/10.1007/s00429-02002046-1

Rockland KS (2020) What we can learn from the complex architecture of single axons. Brain Struct Funct 225(4):21. https://doi. org/10.1007/s00429-019-02023-3 
Rooks B, Anthony M, Chen Q et al (2020) A generic brain connectome map linked to different types of everyday decision-making in old age. Brain Struct Funct 225(4):12. https://doi.org/10.1007/s0042 9-019-02013-5

Rushmore RJ, Bouix S, Kubicki M et al (2020) How human is human connectional neuroanatomy? Front Neuroanat. https://doi. org/10.3389/fnana.2020.00018

Schmahmann JD, Pandya D (2006) Fiber Pathways of the Brain. Oxford Univ Press, New York

Takemura H, Ogawa S, Mezer AA et al (2019) Diffusivity and quantitative $\mathrm{T} 1$ profile of human visual white matter tracts after retinal ganglion cell damage. Neuroimage Clin 23:101826

Thiebaut de Schotten M, Dell'Acqua F, Forkel SJ et al (2011) A lateralized brain network for visuospatial attention. Nat Neurosci 14:1245-1246

Vanderweyen DC, Theaud G, Sidhu J et al (2020) The role of diffusion tractography in refining glial tumor resection. Brain Struct Funct 225(4):24. https://doi.org/10.1007/s00429-020-02056-z

Van Essen DC, Smith SM, Barch DM et al (2013) The WU-Minn Human Connectome Project: an overview. Neuroimage 80:62-79

Wandell BA, Rauschecker AM, Yeatman JD (2012) Learning to see words. Annu Rev Psychol 63:31-53
Wang H, Magnain C, Wang R et al (2018) as-PSOCT: Volumetric microscopic imaging of human brain architecture and connectivity. Neuroimage 165:56-68

Wassie AT, Zhao Y, Boyden ES (2019) Expansion microscopy: principles and uses in biological research. Nat Methods 16:33-41

Woodward A, Gong R, Abe $\mathrm{H}$ et al (2020) The NanoZoomer artificial intelligence connectomics pipeline for tracer injection studies of the marmoset brain. Brain Struct Funct 225(4):19. https://doi. org/10.1007/s00429-020-02073-y

Zilles K, Amunts K (2015) Anatomical basis for functional specialization. In: Uludağ K, Uğurbil K, Berliner L (eds) fMRI: From Nuclear Spins to Brain Functions, vol. 1. Springer, New York, pp 27-66

Zingg B, Hintiryan H, Gou L et al (2014) Neural Networks of the Mouse Neocortex. Cell 156:1096-1111

Publisher's Note Springer Nature remains neutral with regard to jurisdictional claims in published maps and institutional affiliations. 